\title{
Ferromagnetic Transition in One-Dimensional Itinerant Electron Systems
}

\author{
Kun Yang \\ Department of Physics, Florida State University, Tallahassee, Florida 32306
}

(November 13, 2018)

\begin{abstract}
We use bosonization to derive the effective field theory that properly describes ferromagnetic transition in one-dimensional itinerant electron systems. The resultant theory is shown to have dynamical exponent $z=2$ at tree level and upper critical dimension $d_{c}=2$. Thus one dimension is below the upper critical dimension of the theory, and the critical behavior of the transition is controlled by an interacting fixed point, which we study via $\epsilon$ expansion. Comparisons will be made with the Hertz-Millis theory, which describes the ferromagnetic transition in higher dimensions.

Packs numbers: 71.10.Hf,71.10.Pm
\end{abstract}

Ferromagnetic transitions in itinerant electron systems are among the very first examples of quantum phase transitions studied theoretically [1]. In the approach pioneered by Hertz, one decouples the electron-electron interaction using Hubbard-Stratonovish transformation, integrates out the fermionic degrees of freedom, and arrives at a Ginsburg-Landau-Wilson like free energy functional that involves the ferromagnetic order parameter only, which are bosonic degrees of freedom. The quantum nature of the theory lies in the (imaginary) time dependence of the order parameter. This effective bosonic theory, known as the Hertz-Millis theory, was argued to have upper critical dimension $d_{c}=1$, thus the critical behavior of the transition is expected to be mean-field like for both $d=2$ and $d=3[1,2]$. It has been pointed out recently, however, that the procedure of integrating out gapless fermions may lead to subtle singularities in the expansion of the resultant bosonic free energy functional in terms of the order parameter, or its gradients [3]; such singularities may invalidate the power-counting analysis of Hertz and Millis, and change the critical behavior of the transition [3]. The nature of the transition in two- and three-dimensional itinerant electron systems is currently under extensive theoretical study [3].

Comparatively speaking, much less attention has been devoted to the possibility of ferromagnetic transition in one-dimensional (1D) electron systems, until recently. This is in part due to a theorem of Lieb and Mattis [4], which states that the ground state is a singlet for certain classes of one-dimensional models with spin-independent interactions, thus rules out the possibility of ferromagnetism in these models. The existence of ferromagnetic ground states in 1D models (that are not dictated by the Lieb-Mattis theorem) was only established recently through numerical work [5], in which it was also found that the ferromagnetic transition is second-order, thus there is a quantum critical point. On the experimental side, the so-called " $0.7\left(2 e^{2}\right) / h$ " anomaly (or 0.7 anomaly) in the density-dependence of the conductance of a $1 \mathrm{D}$ electron wire has attracted considerable attention; one of the possible interpretations of this anomaly is sponta- neous magnetization of the electrons, which would give rise to plateau-like behavior near $0.5\left(2 e^{2}\right) / h[6]$. This interpretation receives further support from the observation of similar behavior in the presence of magnetic field near crossings of subbands with opposite spin polarization [7]; in this case the corresponding interpretation is spontaneous pseudospin magnetization of the electrons, where the pseudospin index is actually the subband index. Inspired by these developments, a description of the ferromagnetic phase of a 1D electron liquid is developed in Ref. [8], although the critical behavior of the ferromagnetic transition was not studied in that work.

In this paper we study the critical behavior of ferromagnetic transition in 1D. We derive the effective bosonic field theory that describes the transition using Abelian bosonization, and use renormalization group to study the critical behavior. The bosonization method, which is specific to $1 \mathrm{D}$ systems and extremely powerful, allows one to derive the effective bosonic theory in terms of the ferromagnetic order parameter without having to integrate out gapless fermions; thus the resultant theory does not suffer from singularities that the Hertz-Millis theory may encounter. A straightforward power-counting analysis of the theory indicates that the dynamical exponent $z=2$ at the transition (at tree-level), thus the upper critical dimension is $d_{c}=4-z=2$. As a result the theory is below its upper critical dimension in 1D, thus the critical behavior of the transition is controlled by an interacting fixed point. We study the critical behavior using momentum shell renormalization group combined with $\epsilon$ expansion near $d_{c}=2$, at zero as well as low temperature.

Model and Bosonization - Consider the following Hubbard-like Hamiltonian describing interacting electrons in 1D:

$$
H=\sum_{i j \sigma} t_{i j} c_{i \sigma}^{\dagger} c_{j \sigma}+U \sum_{i} n_{i \uparrow} n_{i \downarrow}+\sum_{i j \sigma \sigma^{\prime}} V_{i j}^{\sigma \sigma^{\prime}} n_{i \sigma} n_{j \sigma^{\prime}},
$$

where $t_{i j}$ are the single electron hopping matrix elements, $U>0$ is an onsite repulsion, and $V_{i j}^{\sigma \sigma^{\prime}}$ represents (possibly spin-dependent) further neighbor repulsion; depend- 
ing on the spin dependence of $V$ the system may possess either full Heisenberg (or $\mathrm{O}(3))$ symmetry, or just Ising (or $\mathrm{Z}(2) \times \mathrm{U}(1)$, the latter is responsible for conservation of $\left.S_{z}^{t o t}\right)$ symmetry. It has been shown [5] that in the presence of further neighbor hopping $\left(t_{i j}\right.$ with $\left.|i-j|>1\right)$, the Lieb-Mattis theorem no longer applies and a ferromagnetic ground state is stabilized for large enough $U$, when $V=0$. One can also stabilize the ferromagnetic phase by having $V$ terms that are spin-dependent and ferromagnetic; this possibility has been considered in the context of atoms trapped in 1D optical lattices [9].

One of the most powerful methods of tackling such 1D models is Abelian bosonization [10]. In this scheme, one takes advantage of the fact that in $1 \mathrm{D}$, all particle-hole excitations can be generated by electron density and current operators which satisfy bosonic commutation relations, and expresses both kinetic energy and interaction terms of $H$ in terms of the electron density and current operators. If one keeps terms that are quadratic and with least number of gradients in the density and current operators, one arrives at the familiar Luttinger liquid (LL) Hamiltonian, which describe decoupled spin and charge excitations of the paramagnetic phase [10]:

$$
\begin{aligned}
& H_{L L}=H_{c}+H_{s} \\
& H_{c}=\frac{1}{2 \pi} \int d x\left[\pi^{2} v_{J c} \Pi_{c}^{2}(x)+v_{N c}\left(\partial_{x} \phi_{c}(x)\right)^{2}\right] \\
& H_{s}=\frac{1}{2 \pi} \int d x\left[\pi^{2} v_{J s} \Pi_{s}^{2}(x)+v_{N s}\left(\partial_{x} \phi_{s}(x)\right)^{2}\right] .
\end{aligned}
$$

Here $\phi_{c}(x)$ and $\phi_{s}(x)$ are the charge and spin fields related to the charge and spin densities of the system:

$$
\rho(x)=\frac{1}{\pi} \partial_{x} \phi_{c}(x), \quad S_{z}(x)=\frac{1}{2 \pi} \partial_{x} \phi_{s}(x) ;
$$

while $\Pi_{\alpha}$ are their conjugate fields satisfying

$$
\left[\phi_{\alpha}(x), \Pi_{\alpha^{\prime}}\left(x^{\prime}\right)\right]=i \delta_{\alpha \alpha^{\prime}} \delta\left(x-x^{\prime}\right)
$$

with $\alpha$ being $c$ or $s$. Physically $\Pi_{\alpha}(x)$ represents local charge or spin current. Clearly the velocity parameters $v_{N c}$ and $v_{N s}$ parametrize the energy cost of charge and spin density fluctuations respectively, and are thus proportional to the inverse charge and spin susceptibilities, while $v_{J c}$ and $v_{J s}$ are proportional to the charge and spin stiffness of the system respectively, as they measure the energy cost of charge and spin current fluctuations.

We emphasize that $H_{L L}$ is an approximation of the original electron Hamiltonian Eq. (1) with generic single electron dispersion relation and two-body interaction. For example, a nonlinear term of the form $\int d x \cos \left(\sqrt{8} \phi_{s}\right)$ that describes back scattering of electrons with opposite spins is neglected here. As pointed by Haldane [11], nonlinearity in single-electron dispersion gives rise to terms beyond quadratic order in $\Pi$ and $\phi$, which represent interactions among the bosons. Also the non-locality of electron-electron interaction (due to $V$ for example) leads to non-trivial wave-vector dependence in Fourier space, which gives rise to terms that are quadratic in $\phi$ but involve higher gradients. These terms, however, are $i r$ relevant in the renormalization group sense, at the Luttinger liquid fixed point described by $H_{L L}$ (Eq. (2)); they scale to zero in the long-wave length and low-energy limit; their physical effect is to renormalize the parameters of $H_{L L}$. Thus the long-wave length, low-energy properties of the system are well described by $H_{L L}$, albeit with renormalized parameters. This is the essence of the Luttinger liquid theory $[11,10]$. What we are going to see below however, is that some of the terms that are irrelevant and neglected at the Luttinger liquid fixed point (which describes the paramagnetic phase only) are crucial for the stability of the ferromagnetic phase, as well as the ferromagnetic critical point; they must be retained for a proper description of the ferromagnetic phase as well as the transition. This should not be surprising, as operators irrelevant at one fixed may well be relevant at other fixed points [12].

We now consider approaching the second order phase boundary from the paramagnetic side, by changing parameters in the Hamiltonian. As we approach the critical point, the spin susceptibility $\chi$ diverges; thus $v_{N s} \propto$ $1 / \chi \rightarrow 0$ ! As we move further into the ferromagnetic phase, one expects $v_{N s}$ to become negative; when this happens it becomes energetically favorable to have a nonzero expectation value of $\partial_{x} \phi_{s}(x)$, which is the spontaneous magnetization. Physically this occurs because the gain of exchange energy from the magnetization overcomes the loss of kinetic energy, as is standard in ferromagnetism in itinerant electron systems. Clearly with a negative coefficient for $\left(\partial_{x} \phi_{s}(x)\right)^{2}, H_{s}$ is not stable, and higher order terms in gradients of $\phi_{s}(x)$ and powers of $\partial_{x} \phi_{s}(x)$ must be retained to maintain stability:

$$
H^{\prime}=\int d x\left[a\left(\partial_{x}^{2} \phi_{s}(x)\right)^{2}+b\left(\partial_{x} \phi_{s}(x)\right)^{4}+\cdots\right],
$$

where

in the weak coupling limit $a \approx\left(1 / 8 \pi^{2}\right) \sum_{j} j^{2}\left(V_{n, n+j}^{+-}-V_{n, n+j}^{++}\right)$ and $\left.b \approx \frac{1}{24 \pi} \frac{d^{3} \epsilon(k)}{d k^{3}}\right|_{k=k_{F}}(\epsilon(k)$ is the single electron dispersion). It is clear that the coefficient $a$ is positive for generic repulsive interactions when there is Ising anisotropy $\left(V^{+-}>V^{++}=V^{--}\right)$. On the other hand the sign of coefficient $b$ depends on the details of single electron dispersion; we assume $b$ to be positive here [13], so that the transition is second order which corresponds to the situation in Ref. [5]. Other possible terms that are allowed by symmetry can be shown to be irrelevant at the ferromagnetic critical point to be studied below [14]. Thus combining $H_{s}$ with $H^{\prime}$, switching from Hamiltonian to action which is more convenient for RG analysis, and after rescaling the spin field $\left(\phi=\sqrt{2 a} \phi_{s}\right)$, we arrive at the following effective action: 
$S=\int d \tau d x\left[\frac{1}{2}\left(\partial_{\tau} \phi\right)^{2}+\frac{1}{2}\left(\partial_{x}^{2} \phi\right)^{2}+\frac{r}{2}\left(\partial_{x} \phi\right)^{2}+u\left(\partial_{x} \phi\right)^{4}\right]$

where $\tau$ is imaginary time, $r=v_{N s} /(4 \pi a)$, and $u=$ $b /\left(4 a^{2}\right)$. At mean field level, the ferromagnetic transition occurs at $r=0$. We note that the last three terms in Eq. (8) (or the static parts) take the usual form of Landau theory, upon identifying $\partial_{x} \phi$ as the local magnetization $m(x): \frac{r}{2} m^{2}+\frac{1}{2}\left(\partial_{x} m\right)^{2}+u m^{4}$. The first term in Eq. (8) controls the fluctuations along the imaginary time direction, and is responsible for the quantum nature of the theory. The effective action (8) is the basis of our study of the critical behavior of the ferromagnetic transition, which we now turn to.

Renormalization Group and Critical Behavior at $T=0$ - We perform a renormalization group ( $\mathrm{RG})$ analysis of the action (8), and as is standard in such analysis, we treat the spatial dimension $d$ as a continuous variable, even though the action (8) is derived in $1 \mathrm{D}$. We perform the following space-time transformation with scale factor $s>1: x^{\prime}=x / s, t^{\prime}=t / s^{z}$, and $\phi^{\prime}\left(x^{\prime}\right)=\phi(x) s^{\Delta}$, that leaves the first two terms in (8) invariant. This leads to $z=2$ and $\Delta=d / 2-1$, which in turn lead to the (tree-level) scaling relation for $r$ and $u$ : $r^{\prime}=r s^{2}$ and $u^{\prime}=u s^{2-d}$. We thus find $u$ is relevant below the upper critical dimension $d_{c}=2$, and the critical property of the transition is controlled by an interacting fixed point for $d<2$. Obviously this fixed point is very different from the Luttinger liquid fixed point (which is non-interacting and has dynamical exponent $z=1$ ), even though we are in 1D. In the following we study the critical property using momentum shell RG combined with $\epsilon=2-d$ expansion, and hope it gives a reasonable description at $d=1$.

We assume there is a momentum cutoff $\Lambda$, while no such cutoff exists in frequency. Integrating out modes with $\Lambda / s<k<\Lambda$ and arbitrary frequency at one loop level, we obtain the following flow equations for $r$ and $u$ for small $\epsilon$ :

$$
\begin{aligned}
& \frac{d r}{d \log s}=2 r+\frac{3 u}{\pi}\left(\Lambda^{2}-\frac{1}{2} r\right) \\
& \frac{d u}{d \log s}=\epsilon u-\frac{9}{2 \pi} u^{2} .
\end{aligned}
$$

From these we obtain the fixed point (to order $O(\epsilon)$ ): $u^{*}=(2 \pi / 9) \epsilon$ and $r^{*}=-(\epsilon / 3) \Lambda^{2}$. This interacting fixed point controls the critical property of the transition, at which the RG dimension of the (relevant) tuning parameter $r$ is $y_{r}=2(1-\epsilon / 6)$. From $y_{r}$ as well as the fact that the field $\phi$ receives no anomalous dimension $(\eta=0)$ to order $O(\epsilon)$, we determine the critical exponents: correlation length exponent $\nu=1 / y_{r} \approx(1 / 2)(1+\epsilon / 6)$; susceptibility exponent $\gamma=(2-\eta) \nu \approx 1+\epsilon / 6$; magnetization exponent $\beta=\nu d / 2 \approx 1 / 2-\epsilon / 6$; and field exponent $\delta \approx 3+\epsilon$.
To order $O\left(\epsilon^{2}\right)$ the field $\phi$ receives a non-zero anomalous dimension $\eta$, which also leads to a correction to the dynamical exponent $z=2-\eta / 2[15]$.

RG and Critical Behavior at Finite $T$ - The phase transition occurs at $T=0$ only. However the quantum critical point has very significant influence on the thermodynamic properties at finite $T$, if the system is sufficiently close to the critical point. More specifically, as we will see below, finite temperature introduces a thermal length scale $\xi_{T} \sim T^{-1 / z}$, and depending on its interplay with the correlation length $\xi$ of the system at $T=0$, one can divide the temperature-coupling space into three regions, which are separated from each other by two crossover lines of the form $T \sim\left|r-r^{*}\right|^{z \nu}[16]$ :

(i) Quantum Disordered: $r>r^{*}$ (where $r^{*}$ is the critical coupling) and $\xi_{T}>\xi$, in which the system behaves as an ordinary Luttinger liquid; for example the specific heat $C \sim T$ and susceptibility $\chi \sim$ constant.

(ii) Renormalized Classical: $r<r^{*}$ and $\xi_{T}>\xi$, the system behaves like a ferromagnetic Luttinger liquid [8] whose magnetic order is suppressed by thermal fluctuations; here depending on the symmetry there can be two types of behavior: (a) if the system possesses full Heisenberg symmetry, the gapless transverse spin fluctuation with spectrum $\omega \sim k^{2}$ gives rise to specific heat $C \sim \sqrt{T}$, while the susceptibility $\chi \sim 1 / T^{2}[17]$; (b) if the system possesses Ising symmetry only, then the transverse spin fluctuation is gapped, the longitudinal fluctuation with linear spectrum (the usual Luttinger liquid behavior) gives rise to specific heat $C \sim T$, while the susceptibility $\chi \sim \exp (J / T)$, which is the usual behavior of an Ising ferromagnet ( $J$ is an energy scale of order the domain wall energy of the Ising ferromagnet).

(iii) Quantum Critical: $\xi_{T}<\xi$, in which the thermodynamic property is controlled by the quantum critical fixed point studied above. In the following we focus on the quantum critical region. To study finite $T$ properties, we need to generalize the RG flow equation in the presence of finite temperature. The temperature $T$ determines the range of imaginary time: $0<\tau<1 / T$. Thus under scaling $T$ scales as: $T^{\prime}=T s^{z}$. The flow equation of $r$ is modified to be

$$
\frac{d r}{d \log s}=2 r+\frac{3 u}{\pi}\left(\Lambda^{2}-\frac{1}{2} r\right) \operatorname{coth} \frac{\Lambda^{2}}{2 T},
$$

which leads to a flow away from the fixed point value $r=$ $r^{*}$ due to finite $T$ (flow of $u$ is negligible if initially $u=$ $\left.u^{*}\right)$. Integrating Eq. (11) till $r-r^{*} \sim \Lambda^{2}$, at which scale the system is far away from criticality and the correlation length $\xi(s) \sim 1 / \Lambda$, we find that the temperature $T$ sets a correlation length

$$
\xi_{T}=s \xi(s) \approx \frac{1}{\Lambda \epsilon^{\left(1 / y_{r}\right)}}\left(\frac{T}{\Lambda^{2}}\right)^{-1 / z} \propto T^{-1 / z}
$$

as anticipated. This leads to the temperature dependence of susceptibility $\chi \sim \xi^{2} \sim 1 /(\epsilon T)$ in the quantum 
critical region, to order $O(\epsilon)$. To determine the behavior of specific heat, we need the singular contribution to the free energy from critical fluctuations, which obeys the hyper-scaling law for theories below their upper critical dimensions:

$$
F \sim T^{1+d / z} \Phi\left(\left|r-r^{*}\right|^{z \nu} / T\right),
$$

where $\Phi(x)$ is a universal scaling function. From Eq. (13) we immediately obtain $C \sim T^{1-\epsilon / 2}$ in the quantum critical region.

Summary and Discussion - In this work we developed a bosonic field theory that describes ferromagnetic transition in 1D itinerant electron systems, based on Abelian bosonization. This approach is quite different from that of the Hertz-Millis theory, because in principle the bosonization procedure keeps all the degrees of freedom of the original fermionic system, and allows us to arrive at a bosonic theory without integrating out gapless fermionic degrees of freedom, as was done in the Hertz-Millis theory. We thus believe the theory developed here is free of the possible singularities associated with integrating out gapless fermions.

The bosonic theory developed here, when generalized to arbitrary dimensions, was found to have upper critical dimension $d_{c}=2$. Thus for $d=1$, which is where the theory applies, the system is below its upper critical dimension, and the universal critical behavior of the transition is controlled by an interacting fixed point, which we have studied in some detail using $\epsilon$ expansion. This is again quite different from the Hertz-Millis theory, which is above its upper critical dimension $d_{c}=1$ for $d=2$ and $d=3$ where it applies; there the critical behavior is controlled by a Gaussian fixed point, and some of the critical properties are non-universal due to the presence of dangerously irrelevant operators.

The Abelian bosonization procedure can be applied to systems either with full Heisenberg symmetry or Ising symmetry only. However since it does not exhibit the Heisenberg symmetry explicitly, this symmetry is most likely lost due to the approximate nature of the derivation and treatment of the bosonic theory. Thus the critical behavior discussed above probably applies to systems with Ising symmetry only. In solid state systems the Heisenberg symmetry of electron spins are often reduced to Ising due to the ubiquitous spin-orbit coupling; in systems with pseudospin transitions the Heisenberg symmetry is absent in the first place. Thus the results presented here are highly relevant. Nevertheless it would be highly desirable to maintain the Heisenberg symmetry when present, using for example non-Abelian bosonization, and study if and how the extra symmetry affects critical behavior of the transition. We leave this for future investigation.

In an earlier work, Sachdev and Senthil [18] studied ferromagnetic transitions in lattice rotor models, and arrived at an effective action very similar to Eq. (8); the only difference being the field is complex in their work, which is crucial for the Heisenberg $(\mathrm{O}(3))$ symmetry of their model. If the symmetry were reduced to $\mathrm{Z}(2) \times \mathrm{U}(1)$, the corresponding theory would involve a real field, then the theory becomes identical to Eq. (8) [15]. It was conjectured $[15,18]$ that this action properly describes ferromagnetic transition in 1D itinerant electron systems. It is remarkable that two very different approaches lead to the same effective theory for the transition.

Incidentally, a 2D version of the action (8) was used to study transitions between different valence bond solid states in 2D recently [19]. The physics of these transitions are very different from the one discussed here however.

This work was initiated while the author was visiting the Max Planck Institute for Physics of Complex Systems in Dresden, during her Workshop on Quantum Phase Transitions. He benefited from stimulating discussions with Peter Kopietz. He is particularly grateful to Subir Sachdev and T. Senthil for explaining to him the connection between Ref. [18] and the present work, and bringing Ref. [19] to his attention. This work was supported by NSF grant No. DMR-0225698.

[1] J. A. Hertz, Phys. Rev. B 14, 1165 (1976).

[2] A. J. Millis, Phys. Rev. B 48, 7183 (1993).

[3] See, e.g., D. Belitz and T. R. Kirkpatrick, Phys. Rev. Lett. 89, 247202 (2002); T. R. Kirkpatrick and D. Belitz, Phys. Rev. B 67, 024419 (2003); A. V. Chubukov, C. Pepin, and J. Rech, cond-mat/0311420 (2003).

[4] E. H. Lieb and D. Mattis, Phys. Rev. 125, 164 (1962).

[5] S. Daul and R. M. Noack, Phys. Rev. B 58, 2635 (1998).

[6] K. J. Thomas et al., Phys. Rev. Lett. 77, 135 (1996); D. J. Reilly et al., Phys. Rev. Lett. 89, 246801 (2002).

[7] A. C. Graham et al., Phys. Rev. Lett. 91, 136404 (2003).

[8] L. Bartosch, M. Kollar, and P. Kopietz, Phys. Rev. B 67, 092403 (2003).

[9] H. Pu et al., Phys. Rev. Lett. 87, 140405 (2001).

[10] For a recent review, see, e.g., J. Voit, Rep. Prog. Phys. 58, 977 (1995).

[11] F. D. M. Haldane, J. Phys. C 142585 (1981).

[12] The importance of some of these "irrelevant terms" for the stability of the ferromagnetic phase has been pointed in Ref. [8]. A very similar situation occurs in the field theory description of the reconstructed phase as well as the reconstruction transition at the edge of a quantum Hall liquid, which is an example of chiral Luttinger liquid: K. Yang, Phys. Rev. Lett. 91, 036802 (2003).

[13] If $b$ is negative then higher order terms in $\partial_{x} \phi_{s}(x)$ must be retained, which will lead to a first-order ferromagnetic transition. Only even powers of $\partial_{x} \phi_{s}(x)$ are present as guaranteed by spin symmetry.

[14] In fact there are also non-linear terms of the form $\int d x\left(\partial_{x} \phi_{c}(x)\right)\left(\partial_{x} \phi_{s}(x)\right)^{2}$ etc that actually couple spin 
and charge sectors; since the transition happens in the spin sector, the charge sector can be integrated out which generates terms that are already present in $H_{s}$ and $H^{\prime}$. No singularities are generated here.

[15] S. Sachdev, private communication.

[16] S. Chakravarty, B. I. Haperin and D. R. Nelson, Phys. Rev. B 39, 2344 (1989).

[17] M. E. Fisher, Am. J. Phys. 32, 343 (1964).

[18] S. Sachdev and T. Senthil, Ann. Phys. 251, 76 (1996).

[19] A. Vishwanath, L. Balents, and T. Senthil, condmat/0311085; E. Fradkin, David A. Huse, R. Moessner, V. Oganesyan, and S. L. Sondhi, cond-mat/0311353. 